\title{
Invasives Adenokarzinom der Zervix möglichst aggressiv behandeln?
}

\author{
Bei jeder vierten Patientin mit invasivem Gebärmutterhalskrebs wird ein Adenokarzinom diagnostiziert. Bei \\ diesem Zelltyp ist die Prognose deutlich schlechter als beim Plattenepithelkarzinom. Ein Team aus China testete \\ Wirksamkeit und Toxizität einer aggressiven Behandlungsstrategie.
}

$\mathrm{D}$ ie Studienautoren hatten insgesamt 880 neu diagnostizierte Patientinnen mit einem invasiven Adenokarzinom der Zervix (FIGO-Stadien IIB-IVA) in zwei gleich große Vergleichsgruppen geteilt: Gruppe 1 erhielt eine Radiochemotherapie (concurrent chemoradiation, CCRT). Dabei wurden die Frauen über vier bis fünf Wochen jeweils fünfmal wöchentlich extern mit jeweils 2 Gy bestrahlt, hinzu kamen zweimal wöchentlich intrakavitäre Iridiumapplikationen à $6 \mathrm{~Gy}$. Jeweils zwei Stunden vor oder nach der Bestrahlung wurden zusätzlich Infusionen mit Cisplatin verabreicht. Bei der zweiten Gruppe wurde dieses Regime durch eine adjuvante Chemotherapie verstärkt. An Tag 1 erhielten die Frauen $135 \mathrm{mg} / \mathrm{m} 2$ Paclitaxel, an Tag 2 waren es $75 \mathrm{mg} / \mathrm{m} 2$ Cisplatin. Danach folgte die Radiochemotherapie und eine bzw. drei Wochen später nochmals zwei zusätzliche Zyklen einer Konsolidierungstherapie mit Paclitaxel bzw. Cisplatin.
Mit der alleinigen Radiochemotherapie wurde bei 208 Frauen eine komplette, bei 232 eine partielle Remission erreicht. In Gruppe 2 zeigten 312 Patientinnen vollständiges, 128 partielles Ansprechen. Der Unterschied war signifikant ( $p<0,001)$. Einen Monat nach CCRT-Ende stieg die Zahl der kompletten Responder auf 328 in Gruppe 1 und 378 in Gruppe 2 ( $\mathrm{p}<$ 0,001). In Gruppe 2 waren zusätzlich 42 partielle Responder zu vollständigen Respondern konvertiert. Die intensiver behandelten Patientinnen hatten jedoch deutlich mehr Leuko- und Thrombozytopenien. 215 Patientinnen der Gruppe 2 benötigten Bluttransfusionen, verglichen mit 146 in Gruppe 1. Die Hämoglobinspiegel waren während der Therapie auf einen Tiefpunkt von im Mittel 10,4 (Gruppe 2) bzw. 10,6 (Gruppe 1) gesunken. Nach der symptomatischen Therapie erholten sich aber alle Patientinnen gut. Nach im Mittel 60 Monaten waren 71,4\% der

\section{Auch postmenopausal schützt Sport vor Krebs}

— Bekanntlich trägt regelmäßige körperliche Aktivität dazu bei, das Risiko auch für Brustkrebs zu senken. Nicht ganz klar war bisher, auf welche Lebensphasen und auf welche Intensität es dabei ankommt. Im Long Island Breast Cancer Study Project mit etwa 1.500 Brustkrebspatientinnen und mehr als 1.550 Frauen ohne Brustkrebs in der Kontrollgruppe im Alter zwischen 20 und 98 Jahren wurde diesen Fragen nachgegangen. Die meisten für die Studie befragten Frauen hatten wenigstens ein Kind. Mit Blick auf die Risikoreduktion ergab sich kein Unterschied zwischen der Intensität der körperlichen Aktivität, gemessen in MET (metabolische Äquivalente), und deren Dauer. Im Vergleich zu Frauen, die angaben, nicht regelmäßig körperlich aktiv gewesen zu sein, hatten jene Frauen in der Postmenopause das geringste Brustkrebsrisiko, die sagten, sie wären in der Phase zwischen der Geburt des ersten Kindes und der Menopause mit 10-19 Stunden pro Woche regelmäßig körperlich aktiv gewesen. Mit einer Odds Ratio (OR) von 0,67 war damit das Brustkrebsrisiko um mehr als $30 \%$ verringert. Selbst Frauen, die erst ab der Menopause sportlich aktiver wurden, profitierten davon. Denn wenn sie in diesem Lebensabschnitt zwischen 9 und 17 Stunden pro Woche Sport machten, lag die OR immer noch bei 0,70. Frauen, die jedoch trotz regelmäßigem Sport an Gewicht zulegten (mind. 3 kg), machten damit die erhoffte Krebsprävention zunichte, vor allem wenn die Gewichtszunahme nach der Menopause erfolgte.

Peter Leiner

McCullough LE et al. Fat or fit: The joint effects of physical activity, weight gain, and body size on breast cancer risk. Cancer 2012; online 25. Juni. DOI: 10.1002/cncr.27433

Frauen in Gruppe 2 krankheitsfrei, 60,4\% in Gruppe 1. In Gruppe 2 war der Tumor bei $28,6 \%$ wiedergekehrt, in Gruppe 1 bei $39,6 \%$. Die Zahl der Frauen, die an einem Tumorrezidiv gestorben waren, betrug 113 gegenüber 175. Trotz der hämatologischen Toxizität bewerten die Autoren die neue aggressive Strategie beim Adenokarzinom der Zervix als hoch effektiv und sicher. Die Compliance war trotz verlängerter Therapiedauer gut. Doch gilt es zu bedenken, dass fast die Hälfte der Frauen, die zusätzlich zur Radiochemotherapie eine adjuvante Chemotherapie erhalten hatten, mindestens einer Bluttransfusion bedurften. Aber: „Die sinkenden Hämoglobinspiegel haben das Überleben der Patientinnen offensichtlich nicht negativ beeinflusst", so die Autoren.

Dr. Elke Oberhofer

Tang J et al. Chemoradiation and adjuvant chemotherapy in advanced cervical adenocarcinoma. Gynecol Oncol 2012; 125: 297-302 doi:10.1016/j.ygyno.2012.01.033

Kommentar: Der histologische Typ eines Adenokarzinoms der Zervix ist mit einer deutlich verschlechterten Prognose verbunden. Trotzdem unterscheidet sich bislang die Therapie eines Adenokarzinoms leitlinienkonform nicht mit der eines Plattenepithelkarzinoms. So wird bislang grundsätzlich die Durchführung einer adjuvanten Chemotherapie beim Zervixkarzinom, unabhängig von dem histologischen Typ, abgelehnt. In der vorgelegten prospektiv randomisierten Studie konnte nun erstmalig belegt werden, dass durch eine zusätzlich zu einer konventionellen Radiochemotherapie durchgeführte neoadjuvante/adjuvante Kombinationschemotherapie der klinische Erkrankungsverlauf signifikant verbessert werden kann. Somit steht dieser Studie zufolge bei einem Hochrisikokollektiv von Zervixkarzinom-Patientinnen, definiert durch den histologischen Typ eines Adenokarzinoms und ein fortgeschrittenes Tumorstadium, eine neue therapeutische Option in Form einer Chemotherapie zur Verfügung.

Prof. Dr. Peter Mallmann 